\title{
Toward a User Acceptance Model of Autonomous Driving
}

\author{
Konstantin Garidis \\ Reutlingen University \\ konstantin.garidis@ \\ reutlingen-university.de
}

\author{
Leon Ulbricht \\ Reutlingen University \\ ulbricht.leon@ \\ googlemail.com
}

\author{
Alexander Rossmann \\ Reutlingen University \\ alexander.rossmann@ \\ reutlingen-university.de
}

\author{
Marco Schmäh \\ Reutlingen University \\ marco.schmaeh@ \\ reutlingen-university.de
}

\begin{abstract}
Autonomous driving is becoming the next big digital disruption in the automotive industry. However, the possibility of integrating autonomous driving vehicles into current transportation systems not only involves technological issues but also requires the acceptance and adoption of users. Therefore, this paper develops a conceptual model for user acceptance of autonomous driving vehicles. The corresponding model is tested through a standardized survey of 470 respondents in Germany. Finally, the findings are discussed in relation to the current developments in the automotive industry, and recommendations for further research are given.
\end{abstract}

\section{Introduction}

The technology of autonomous driving is becoming the next big digital disruption in the automotive industry. With fully autonomous driving, a technical system takes over the control of the vehicle, completely replacing the human as the driver of the automobile. Experts propose that self-driving cars will generate immense benefits for individuals and society, including greater traffic safety, better fuel economy and higher time savings [14]. Furthermore, specialists expect that driverless cars will create a completely new traffic system, which not only comes with new possibilities for traffic control but also generates completely new transport offers [16]. For example, the idea that the time spent in the vehicle does not need to be spent on driving requires a complete re-evaluation of mobility. Accordingly, the car will no longer be a pure means of transport but will become a third living space for users. For this reason, the way the interior of an autonomous car is designed and operates will also gain in importance [14].

In addition to all these benefits of driverless cars however, experts are also calling attention to the immense legal and ethical challenges that come with this disruptive technology and which will strongly influence the integration of autonomous cars into the global transportation system [16]. Moreover, the possibility of fully integrating autonomous vehicles into current transport systems not only involves the vehicle technology itself but also requires the acceptance and adoption of users. Although traditional car manufacturers, automotive suppliers and tech companies are heavily investing in the autonomous driving technology, the question is whether the automotive market and consumers are ready for this technology. That is, while autonomous driving is currently one of the most debated technologies in the automotive industry, customer acceptance is still not sufficiently researched.

Although some research has examined user acceptance of autonomous cars, only a few studies have applied theoretical or conceptual models of acceptance based on empirical data [10]. Therefore, this thesis aims to close this research gap by examining the factors that might influence the adoption and acceptance of self-driving cars by applying the unified theory of acceptance and use of technology (UTAUT) model in conjunction with a quantitative questionnaire [27]. In addition, this paper aims to derive non-drivingrelated activities that users would like to perform while riding in an autonomous vehicle. In this case, the car turns into a third place of living beyond the traditional places of private homes and working environments.

Coherent with these research objectives, two research questions are addressed in this study: (1) Which factors influence the user acceptance of autonomous driving vehicles? and (2) Which activities would people perform while riding in an autonomous vehicle? In line with these questions, a conceptual model for user acceptance of autonomous driving vehicles is developed. The corresponding model is tested through a standardized survey of 470 respondents in Germany. Furthermore, this paper aims to assess non-driving-related activities of autonomous car users in the third place. Empirical findings provide insights into the antecedents for intentions to use an autonomous driving vehicle. In addition, the use case evaluation of the third place might guide interior design strategies of manufacturers of self-driving cars. 


\section{Theoretical Background}

With the growing attention to autonomous driving, researchers and car manufacturers are striving for a consistent definition of autonomous driving. According to various authors [1], the term "autonomous" means having the power to self-govern. According to this, driving autonomously entails the independent and targeted driving of a vehicle in real traffic situations without the intervention of a driver [13].

Throughout the paper, terms such as "self-driving" and "fully automated vehicles" are used as synonyms for autonomous vehicles. However, even if basic definitions of autonomous driving all focus on the similar characteristics of an autonomous car, the Society of Automotive Engineers (SAE) is working to provide a common understanding of the term by defining five different classifications of automation levels, ranging from "No Driving Automation" (0) to "Full Driving Automation" (5). Levels 0-2 classify cars as using assistant technologies such as object detection and collision control, but the driver is still the main operator of the vehicle. Similarly, levels 3 and 4 represent autonomous cars that can drive autonomously, but the driver still needs to be ready as a fallback to take control. Level 5 vehicles are fully automated and do not require a driver at all [25].

This paper additionally investigates the evaluation of use cases in the third place. The term "third place" is of particular significance and needs to be defined more precisely. Oldenburg [20] describes "third places" as informal public gathering places, such as cafés, pubs, and libraries. Thus, they differ from a person's home, which is considered the first place in life, and the work environment, which is viewed as the second place [20]. In the context of autonomous driving, the car is regarded as the third place, becoming another essential area outside the home and office.

In the past few years, studies on the acceptance of autonomous driving technology have evolved. The current study selected studies on SAE levels 4 and 5 of driving automation, to obtain comparable results of high and full automation. Furthermore, the review is limited to peer-reviewed studies published in English. Table 1 classifies the studies according to research method, location, number of respondents, general level of acceptance, and so on.

Most studies have also been carried out in highincome Western countries. Furthermore, most user acceptance studies have taken place in the United States, possibly because the country was one of the first to recognize and explore the potential of autonomous driving technology. In the most recent study, Hein et. al. [10] conducted their survey in Germany using a marketing agency. The overall level of acceptance in the studies varies significantly, and therefore, no similarities or trends can be derived. A majority of the studies evaluated the acceptance level by asking respondents about their intention to use, willingness to buy and positive impressions. In some studies $[15,23,24]$ respondents were asked to rate their technology acceptance on a scale. Bansal et al. [3] and Payre et al. [23] evaluated willingness to pay under the assumption that consumers are willing to use selfdriving vehicles. These studies evaluated either a specific value or a range at which respondents were willing to pay for fully autonomous driving features. The willingness to pay mostly varied between 1,000 and 5,000 USD.

In their study, Rödel et al. [24] used the car technology acceptance model of Osswald et al. [21], which extends the Technology acceptance model. While they do not explicitly focus their survey on cars of automation level 4 or 5, they also took the expected user experience into account. Several studies [5, 17, 19] partly used the UTAUT model as a framework to gain a better understanding of the factors affecting the construct "intention to use". Hein et al. [10] made use of the technology acceptance model to assess users' adoption of autonomous cars while integrating moderating factors into their model. They found that the instrumental benefits of "work", "internal socializing", "external socializing", "entertainment" and "reading" have significant influences on the perceived usefulness of driving an autonomous car.

In summary, the literature provides inconsistent results on the definition of the user acceptance of autonomous cars. Most studies are of exploratory character that looked into general acceptance and don't use methods that measure explicit user acceptance effects. Therefore, this study focuses on an extensive examination of what influences people's acceptance of using autonomous cars. This is achieved by making use of UTAT, as extensive user acceptance model in current research. In addition, to further complement the current research, this study fills the research gap on which tasks users would like to perform while riding in an autonomous car and their influence on acceptance.

\section{Conceptual Model}

The second version of the UTAUT model [27], serves as a basis for the proposed research model in this paper. Several core constructs of the UTAUT2 model represent an integral part of the research topic and are complemented by constructs that have previously been identified in literature. Given the limited amount of space for conference papers, we focused on the main theoretical foundation of each relevant construct and hypothesis. 
Table 1 Overview of Studies on User Acceptance of Autonomous Cars

\begin{tabular}{|c|c|c|c|c|c|}
\hline Authors & Title & Method & Location & Sample & $\begin{array}{l}\text { General Level of } \\
\text { Acceptance }\end{array}$ \\
\hline Payre et al. 2014 & $\begin{array}{l}\text { Intention to use a fully automated car: attitudes } \\
\text { and a priori acceptability }\end{array}$ & $\begin{array}{l}\text { Interview/paper- } \\
\text { based survey/ } \\
\text { online survey }\end{array}$ & France & $5 / 45 / 421$ & $\begin{array}{l}\text { General acceptance: } \\
68.1 \% \text { above } 4 / 7 \text { on a } \\
\text { scale }\end{array}$ \\
\hline Rödel et al. 2014 & $\begin{array}{l}\text { Towards autonomous cars: the effect of } \\
\text { autonomy levels on acceptance and user } \\
\text { experience }\end{array}$ & Online survey & Austria & 336 & $\begin{array}{l}\text { Intention to use: } \\
3.04 / 6\end{array}$ \\
\hline Bazilinskyy et al. 2015 & $\begin{array}{l}\text { An international crowdsourcing study into } \\
\text { people's statements on fully automated driving }\end{array}$ & $\begin{array}{l}\text { Three online } \\
\text { surveys }\end{array}$ & 112 countries & 8.862 & $\begin{array}{l}\text { Positive attitude } \\
\text { toward automated } \\
\text { driving: } 39 \%\end{array}$ \\
\hline Choi and Ji 2015 & $\begin{array}{l}\text { Investigating the importance of trust on adopting } \\
\text { an autonomous vehicle }\end{array}$ & Survey & South Korea & 552 & $\mathrm{n} / \mathrm{a}$ \\
\hline Kyriakidis et al. 2015 & $\begin{array}{l}\text { Public opinion on automated driving: results of } \\
\text { an international questionnaire among } 5000 \\
\text { respondents }\end{array}$ & Online survey & 109 countries & 4.886 & $\begin{array}{l}\text { Enjoyable mean: } \\
3.49 / 5 \text { on a scale }\end{array}$ \\
\hline Bansal et al. 2015 & $\begin{array}{l}\text { Assessing public opinions of and interest in new } \\
\text { vehicle technologies: an Austin perspective }\end{array}$ & Online survey & Austin, Texas & 347 & $\begin{array}{l}\text { Interest in having } \\
\text { level } 4 \text { AVs: } 81 \%\end{array}$ \\
\hline $\begin{array}{l}\text { Benleulmi and Blecker } \\
2017\end{array}$ & $\begin{array}{l}\text { Investigating the factors influencing the } \\
\text { acceptance of fully autonomous cars }\end{array}$ & Online survey & USA/Germany & 313 & $\mathrm{n} / \mathrm{a}$ \\
\hline Madigan et al. 2017 & $\begin{array}{l}\text { What influences the decision to use automated } \\
\text { public transport? Using UTAUT to understand } \\
\text { public acceptance of automated road transport } \\
\text { systems }\end{array}$ & $\begin{array}{l}\text { Experiment } \\
\text { questionnaire } \\
\text { survey }\end{array}$ & Greece & 315 & $\mathrm{n} / \mathrm{a}$ \\
\hline Nordhoff et al. 2018 & $\begin{array}{l}\text { Acceptance of driverless vehicles: results from a } \\
\text { large cross-national questionnaire study }\end{array}$ & Online survey & 116 countries & 7.755 & $\mathrm{n} / \mathrm{a}$ \\
\hline Hein et al. 2018 & What drives the adoption of autonomous cars? & $\begin{array}{l}\text { Interview/online } \\
\text { survey }\end{array}$ & Germany & $16 / 643$ & $\begin{array}{l}\text { Adoption intention } \\
\text { mean } 4.47 / 7\end{array}$ \\
\hline
\end{tabular}

The construct performance expectancy is the strongest predictor of behavioral intentions and has a positive influence on the intention to use [27]. The positive effect of performance expectancy on the acceptance of autonomous vehicles has been investigated and validated in various articles [19, 24].

Hypothesis 1: Performance expectancy has a positive effect on behavioral intentions.

The construct effort expectancy gives valuable insights into the perceived difficulty in using autonomous driving systems and has a positive influence on intention to use. The impact of effort expectancy plays a significant role in the context of autonomous vehicle acceptance $[5,19]$.

Hypothesis 2: Effort expectancy has a positive effect on behavioral intentions.

Social influence exerts an impact on drivers' individual behaviors through compliance, internationalization and identification. In many cases, cars and their specific technology are perceived as status symbols. The identification with autonomous vehicles can be advanced through the link between acceptance and social influence $[5,17,21]$.

Hypothesis 3: Social influence has a positive effect on behavioral intentions.

Hedonic motivation, also referred to as perceived enjoyment, is an important construct that can be used to predict consumers' intentions to use an autonomous vehicle. Madigan et al. [17] found that hedonic motivation was the strongest predictor of intention to use. Other studies have also explored the relevance of hedonic motivation in the autonomous driving context $[5,17,21]$.

Hypothesis 4: Hedonic motivation has a positive influence on behavioral intentions.

The price-value ratio of autonomous vehicles is regarded as a critical factor for user acceptance. The low willingness to pay for autonomous vehicle systems indicates cost as an area of concern. However, the price-value of autonomous driving is likely to positively influence the intention to use autonomous driving vehicles [5]. 
Hypothesis 5: An attractive price-value evaluation has a positive effect on behavioral intentions.

Many studies regard environmental friendliness of autonomous vehicles as a major benefit. In the study of Schoettle and Sivak [26], survey participants perceived less traffic congestion, lower vehicle emissions and better fuel economy as major benefits of autonomous driving. These findings are also in line with those of Bansal et al. [3].

Hypothesis 6: Environmental friendliness has a positive effect on behavioral intentions.

Desire for control is also a significant factor influencing the acceptance of autonomous vehicles. Respondents consistently express concern about the idea of handing over complete control to the vehicle. More than $90 \%$ of respondents in the surveys of Schoettle and Sivak [26] still wanted to have the power of the steering wheel plus gas and brake availability to control the fully autonomous vehicle when desired.

Hypothesis 7: Desire for control has a negative effect on behavioral intentions.

Loss of driving pleasure is another construct added to the model. Some respondents fear the loss of driving pleasure when manual steering devices disappear and the system takes full control of all driving activities. Respondents in the studies of Bazilinskyy et al. [4], Kyriakidis et al. [15] and Rödel et al. [24] preferred manual driving to automated driving because of the "joy of driving".

Hypothesis 8: Loss of driving pleasure has a negative effect on behavioral intentions.

Safety is the most significant factor driving the acceptance of autonomous vehicles and is perceived as both a facilitator to and an obstacle of autonomous vehicles. For example, Schoettle and Sivak [26] showed that respondents expected autonomous vehicles to help reduce car crashes. On the other side, even more respondents are worried about safety consequences of equipment failure or system failure. However, in the majority of the reviewed studies, respondents predominantly perceived safety as the greatest concern. The perceived level of safety is expected to positively influence behavioral intentions.

Hypothesis 9: Safety has a positive effect on behavioral intentions.

Security of the system and the vehicle is another important construct. Respondents consistently expressed concerns about vehicle and system security. Respondents in the survey of Hein et al. [10] rated security especially in terms of hacking and data theft as a concern. Therefore, security is expected to positively correlate with the intention to use.

Hypothesis 10: Security has a positive effect on behavioral intentions.
Data privacy is another topic of concern, with study results showing that people are worried about the misuse of their personal data. Autonomous driving enables non-stop location and destination tracking, as well as access to other users' data, which are stored in the cloud, leading to respondents' fear of misuse [15, 26]. Therefore, the perceived level of data privacy is expected to positively correlate with the intention to use.

Hypothesis 11: Data privacy has a positive effect on behavioral intentions.

The legal situation with regard to autonomous vehicles is another topic of respondent concern. Respondents of the reviewed studies expressed high levels of concerns about current legal regulations [15, 22]. In the survey of Schoettle and Sivak [26], threequarters of the respondents expressed concerns about legal liabilities for drivers due to insufficient governmental regulations.

Hypothesis 12: A structured and clear legal situation has a positive effect on behavioral intentions.

Trust and its influencing factors have been an integral part of the evolution of technology acceptance models [2, 28]. Mayer et al. [18], p. 712 define trust as the "willingness of a party to be vulnerable to the actions of another party based on the expectation that the other will perform a particular action important to the trustor, irrespective of the ability to monitor or control that other party". Studies have investigated and proved that trust is a redundant determinant to predicting the acceptance of autonomous driving [5, 19]. Therefore, trust items are included in the construct of behavioral intentions.

Building on the constructs of the UTAUT model [27] and an acceptance of fully autonomous driving, Figure 1 also illustrates the proposed conceptual model.

\section{Method}

We conducted an online survey using the software Unipark for this research. In total, 470 respondents (48\% male, Mage $=43$ years, $29 \% 18-29$ years) were surveyed with the assistance of a market research provider. A qualifying filter question was included to identify and eliminate respondents who were inattentive in completing the survey.

The questionnaire consisted of four different parts and included 50 items. As the survey was conducted in Germany, the whole questionnaire was in German. The average response time per survey respondent was approximately 11 minutes. The purpose of filter questions is to ensure the desired sample of the survey; thus, respondents who are not qualified or experienced 
enough to answer the questionnaire are sorted out. The respondents of this study needed to meet the following characteristics: owned a driver's license, owned or used a car and had basic knowledge of autonomous driving. The second part of the questionnaire contained demographic and personal survey questions on a multiple-choice basis. The demographic questions collected data on age, gender, education and income. The next parts of the survey contained the UTAUT items, and each construct was verified by three items on a 7-point Likert scale ( 1 = "strongly disagree", 7 = "strongly agree"). The only exceptions were the constructs "price", which had two items, and "intention to use", which had five items.

The sample collection was carried out in two stages. First, $40 \%$ of the respondents were randomly collected, and second, $60 \%$ were collected through defined quota parameters. These quota parameters include the relevance of autonomous driving technology for specific age groups. The following quota parameters for the sample size were given: $30 \%$ for those aged of 18 to 29 years and $30 \%$ for those aged 30 to 39 years.

Before the full-scale survey was conducted, the questionnaire was first completed by a small sample of respondents to identify potential problems or inconsistencies in the questionnaire. The pre-test was conducted with 36 respondents to examine the comprehensibility, quality and duration of the questionnaire. From these results, some corrective actions were taken. In addition, an initial evaluation of the pre-test data showed that some constructs of the research model were not significant enough to test the different hypotheses. As a result, the less meaningful items were replaced with stronger ones.

The program SmartPLS served to analyze the given statistical data and to verify the defined research model of the study. Subsequent to the estimation of the parameters, a variance analytical model that calculates the relationships and dependencies between indicators and the latent variables can be constructed $[9,11]$. As a result, PLS-SEM was considered a suitable method to examine the relationships between the single constructs of the research model.

In addition to the analysis of the conceptual model of user acceptance, a specific set of use cases for typical user activities in the third place was tested. In particular, typical user activities while sitting in an autonomous driving car were collected and grouped into four modes. The preferences of the users with regard to the four conceptualized modes were also assessed in the standardized survey.

\section{Result}

Before analyzing the causal model, the measurement models were assessed for the validity and reliability of the conceptualized constructs and items. For consistency purposes, the well-established guidelines of Hair et al. [9] are applied.

\subsection{Measurement Model Assessment}

The internal consistency reliability evaluates the consistency of results obtained with different test items. Traditionally, Cronbach's alpha is used as a measurement metric. However, given the limitations of Cronbach's alpha, composite reliability (CR) is applied. All values in the applied measurement model range between .70 and .95 , thus supporting internal consistency reliability (see Table 2 ).

To evaluate the convergent validity of a reflective construct, the indicator loadings and the average variance extracted (AVE) need to be assessed. The majority of the indicator loadings are clearly higher than the recommended threshold of .7. Only two items have values ranging between .4 and .7. In general, indicators with loadings between .4 and .7 should only be removed from the model if their elimination leads to an increase in CR. Nevertheless, the impact on content validity also must be considered [9] Therefore, the indicators SI2 and DC1 are kept in the model. Assessment of the AVE reveals that all registered values are higher than the required threshold of .5 , suggesting that the construct explains more than half the variance of its indicators on average.

Discriminant validity describes the extent to which a construct differs from other constructs along empirical standards. Traditionally, researchers have relied on two test criteria: cross-loadings and the Fornell-Larcker criterion. However, recent studies have shown that the performance of neither crossloadings nor the Fornell-Larcker criterion is truly reliable. Therefore, Henseler et al. [12] introduced the heterotrait-monotrait (HTMT) ratio of correlations, which is mean of all indicator correlations measuring different constructs in relation to the geometric mean of the average indicator correlation measuring its own construct [9].

According to research, a threshold of .90 is acceptable if the path model contains constructs that are conceptually similar. If the constructs in the path model are conceptually more different, a limit of .85 should be taken. Evaluation of the model shows that all HTMT values are .85 and lower. Thus, all constructs are empirically different. 
Table 2 Validity and reliability of construct items

\begin{tabular}{|c|c|c|c|c|c|}
\hline Construct & Item & Item Description & Loadings & CR & AVE \\
\hline \multirow[t]{5}{*}{$\mathrm{BI}$} & BI1 & I would like to own an autonomous car. & .92 & .92 & .72 \\
\hline & $\mathrm{BI} 2$ & I would use an autonomous car as soon as it is available on the market. & .88 & & \\
\hline & $\mathrm{BI} 3$ & I can imagine the use of an autonomous car-sharing service. & .72 & & \\
\hline & $\mathrm{BI} 4$ & I would trust the driving skills of an autonomous car more than my own. & .80 & & \\
\hline & BI5 & I am highly confident in an autonomous driving system. & .90 & & \\
\hline \multirow[t]{3}{*}{$\mathrm{DC}$} & DC1 & I want to be able to take control of the autonomous car at all times. & 64 & .84 & 64 \\
\hline & $\mathrm{DC} 2$ & The transfer of control to an autonomous car is difficult for me. & .89 & & \\
\hline & DC3 & Being able to control a car manually is important to me. & .83 & & \\
\hline \multirow[t]{3}{*}{ DPR } & DPR1 & I think that data collected on me will not be used for commercial purposes. & .88 & .91 & .78 \\
\hline & DPR2 & The data collected on me will be treated confidentially. & .87 & & \\
\hline & DPR3 & I think that legal regulations will lead to sufficient data protection. & .88 & & \\
\hline \multirow[t]{3}{*}{ EE } & EE1 & I think it would be easy for me to learn to operate an autonomous car. & .80 & .88 & .72 \\
\hline & EE2 & I imagine the handling of an autonomous car to be clear and understandable. & .88 & & \\
\hline & EE3 & I imagine the operation of an autonomous car to be easier than that of a conventional car. & .85 & & \\
\hline \multirow[t]{3}{*}{$\mathrm{EF}$} & EF1 & Autonomous cars lead to a lower traffic load. & .83 & .89 & .73 \\
\hline & EF2 & Autonomous cars have increased fuel efficiency. & .86 & & \\
\hline & EF3 & Autonomous cars are more environmentally friendly than conventional cars. & .86 & & \\
\hline \multirow[t]{3}{*}{$\mathrm{HM}$} & HM1 & Autonomous cars are more entertaining than traditional cars. & .88 & .94 & .84 \\
\hline & HM2 & Using an autonomous car would increase my driving experience. & .92 & & \\
\hline & HM3 & Autonomous cars would give me great pleasure. & .94 & & \\
\hline \multirow[t]{3}{*}{ LDP } & LDP1 & In a car, I prefer being the driver rather than the passenger. & .87 & .91 & .77 \\
\hline & LDP2 & I like driving a car. & .87 & & \\
\hline & LDP3 & The control of a car gives me pleasure. & .89 & & \\
\hline \multirow[t]{3}{*}{ LEG } & LEG1 & I think the current legislation regarding autonomous cars is sufficient. & .83 & .91 & .78 \\
\hline & LEG2 & In the case of an accident of an autonomous car, it is clear who bears the blame. & .89 & & \\
\hline & LEG3 & The legal framework regarding autonomous cars is clearly defined. & .93 & & \\
\hline \multirow[t]{2}{*}{$\mathrm{P}$} & $\mathrm{P} 1$ & I'm ready to pay extra for an autonomous car. & .96 & .95 & .92 \\
\hline & P2 & The benefits of an autonomous car will justify the price. & .95 & & \\
\hline \multirow[t]{4}{*}{$\mathrm{PE}$} & PE1 & Autonomous cars are faster and more efficient than traditional cars. & .77 & .89 & .68 \\
\hline & PE2 & Autonomous cars give me time for other activities. & .86 & & \\
\hline & PE3 & $\begin{array}{l}\text { Autonomous cars enable increased mobility for specific target groups (e.g. minors, elderly } \\
\text { physically disabled persons). }\end{array}$ & .78 & & \\
\hline & PE4 & Driving in an autonomous car increases my productivity. & .88 & & \\
\hline \multirow[t]{3}{*}{ SAF } & SAF1 & Autonomous cars increase traffic safety. & .92 & .94 & .83 \\
\hline & SAF2 & I think that autonomous cars will reduce traffic accidents. & .94 & & \\
\hline & SAF3 & An autonomous car drives more safely than I do. & .88 & & \\
\hline \multirow[t]{3}{*}{ SEC } & SEC1 & I think autonomous driving systems are safe and cannot be hacked. & .92 & .93 & .82 \\
\hline & SEC2 & Autonomous driving systems are not prone to unauthorized third-party access. & .88 & & \\
\hline & SEC3 & I think the cyber security of an autonomous car is guaranteed. & .91 & & \\
\hline \multirow[t]{3}{*}{ SI } & SI1 & My family and friends would like it if I used an autonomous car. & .90 & .83 & .63 \\
\hline & SI2 & $\begin{array}{l}\text { I would like to have my family and friends use an autonomous car first before deciding on it } \\
\text { myself. }\end{array}$ & .51 & & \\
\hline & $\mathrm{SI} 3$ & People who are close to me would encourage me to use an autonomous car. & .91 & & \\
\hline
\end{tabular}

\subsection{Causal Model}

The final structural model provides a detailed overview of the significance of the relationships and the model's predictive power. The model explains $82 \%$ of the variance in intention to use autonomous vehicles and therefore has substantial explanatory power (see Figure 1).

In total, eight of the 12 hypotheses are supported, while four are rejected. The strongest predictor of the UTAUT2 turned out to be non-significant. Moreover, of the seven added predictors based on in-depth literature research, four have a significant effect on intention to use.
The analysis of the path coefficients confirmed the significant, positive effects of safety $\left(.289^{* * *}\right)$ and hedonic motivation $\left(.228^{* * *}\right)$, in support of $\mathrm{H} 9$ and H4. Furthermore, the hypothesized significant, negative effect of desire for control $(-.180 * * *)$ was confirmed, providing support for H7. Likewise, social influence $\left(.116^{* * *}\right)$, price-value $\left(.136^{* * *}\right)$, and security $\left(.107^{* * *}\right)$ all had a significant, positive effect on behavioral intention. Therefore, $\mathrm{H} 3, \mathrm{H} 5$, and $\mathrm{H} 10$ are also accepted. A smaller but also significant, positive effect was found for effort expectancy $\left(.048^{* *}\right)$ and data privacy $\left(.047^{*}\right)$. Thus, H2 and H11 are confirmed. In contrast with expectations, the path analysis did not confirm the hypothesized significant, positive effects for performance expectancy (.050), 
environmental friendliness (-.015) or legal (-.005). Thus, H1, H6, and H12 are not supported. Finally, the hypothesized significant, negative effect of loss of driving pleasure was not significant, rejecting $\mathrm{H} 8$.

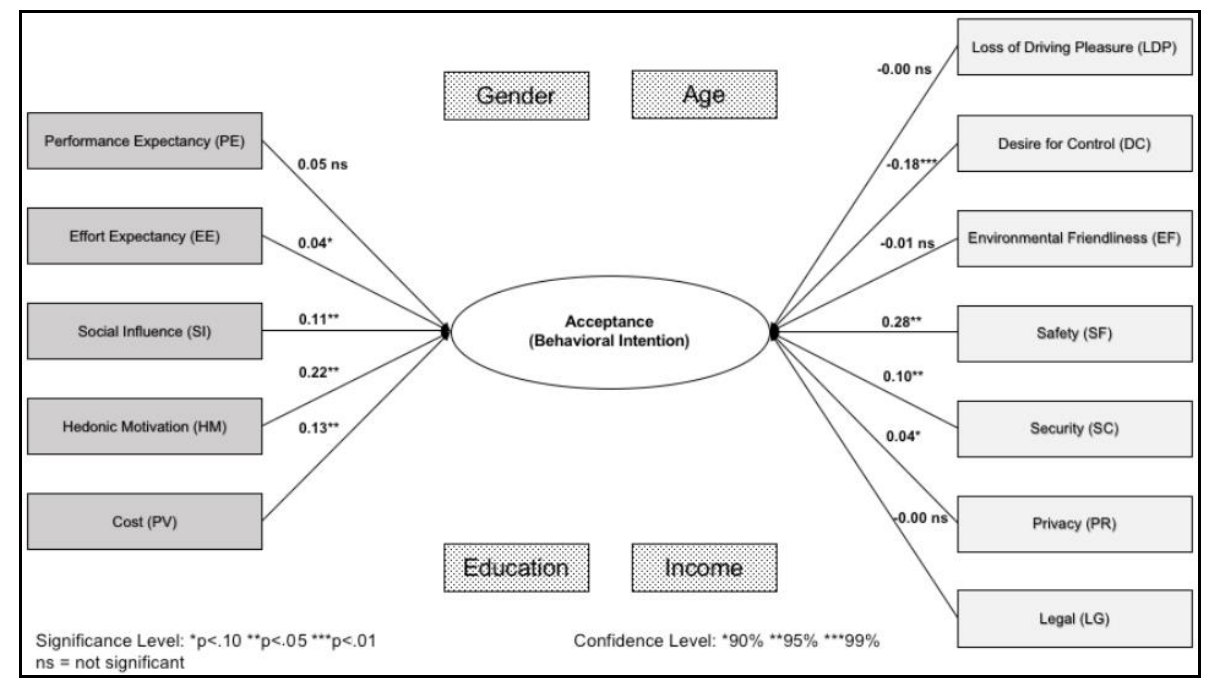

Figure 1 Causal model and p-values

The effect of the four conceptualized control variables was assessed with multi-group analysis. Moderating effects were conceptualized as age, gender, education and income. First, the moderating effect of age was examined. The questionnaire offered a choice of six age groups. For evaluation purposes of the moderating effects, these age groups were subdivided into three groups: young respondents (18-39 years), middle-aged respondents (40-59 years), and old respondents (above 60 years). Subsequently, these age groups were compared with one another. One significant difference was between young and old respondents. Vehicle security and protection against unauthorized interference had no influence on the intention to use for older participants. For younger participants, however, a strong positive effect was found. Second, significant moderating effects were assessed for gender. The only significant difference between women and men was the effect of safety on the intention to use. Although safety plays an important role for both genders, the positive influence on behavioral intention was significantly greater for men. The moderating effects of gender in combination with age were also evaluated. For this purpose, both genders between and within their same age groups were compared. The comparison of women in different age groups shows one significant difference. The direct positive effect of vehicle security on behavioral intentions was significant greater for young than old women. The comparison of men in different age groups also includes some significant deviations. The hedonic motivation of technology had a greater impact on usage intentions for old than young men. However, the social influence of their environment had a significantly stronger impact on the intention to use for young than old men. The comparison of gender within the same age groups did not reveal any significant differences. Third, the moderating effect of education was evaluated. Again, there were seven different answer categories in the questionnaire, which were summarized for evaluation according to the following three categories: education 1 (no school qualification, "Certificate of secondary education"), education 2 (general higher education entrance qualification), and education 3 (bachelor's degree, master's/diploma degree, doctoral degree). The comparison of the different education categories showed significant differences between respondents with a low educational level and those with a median educational level. The significant, negative effect of desire for control on behavioral intentions for those in education 2 was greater than that for people with lower education. However, general safety aspects had a stronger positive influence on the behavioral intentions of people in education 1. Fourth, respondent income was examined as the last moderating effect. The possible seven answer categories of the questionnaire were grouped into three categories: low income (up to 30,000 gross annual salary), middle income (30,000 to 50,000 gross annual salary) and high income (more than 50,000 gross annual income). There was a significant difference between the low-income category and the high-income category. The positive effect of the price-value ratio on behavioral intentions was significantly greater for respondents with high incomes than for those with low incomes. 


\subsection{Third Place}

The evaluation of the questionnaire items shows that of the four proposed interior modes of current conceptual studies, the driving mode received the highest approval at $59 \%$. Family mode received $41 \%$ approval, and $37 \%$ indicated their intention to use the lounge mode. Only $18 \%$ of the respondents indicated an intention to use the business mode. The second interior item dealt with the evaluation of non-drivingrelated activities people would like to perform while riding in an autonomous vehicle. Notably, $69 \%$ of the respondents indicated a desire to look out the window. Figure 2 depicts the activities respondents would like to engage in while riding in an autonomous vehicle. It is organized in four categories of possible activities that the users could engage in their third place of life.

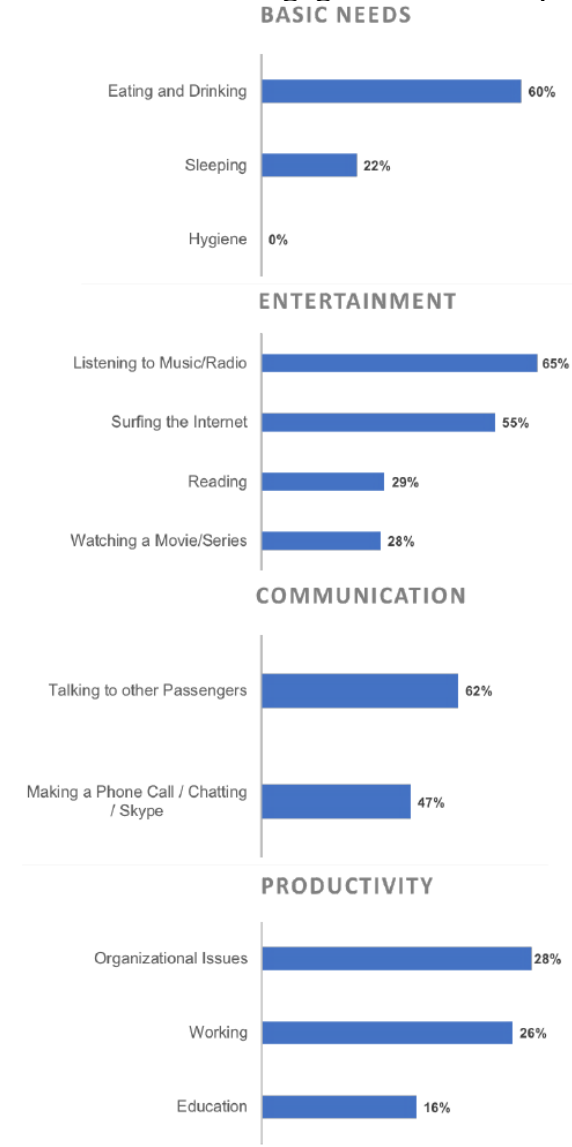

Figure 2 The impact of autonomous cars on customer use of time

\section{Implications}

The findings of the research model provide empirical support for eight of the 12 proposed relationships. The model has substantial predictive power, explaining more than three-quarters of the variance in intentions to use autonomous vehicles. This leads to multiple implications for theory and practice.

This research makes a significant contribution to both user acceptance research in general and the research on driverless autonomous cars in particular. Recent studies are quite vague regarding general user acceptance or show inconsistent results on how user adoption is conceptually defined. This study adds a more detailed account of the factors that drive people to use autonomous vehicles. Further analysis could provide even more detailed insights into how moderating factors could influence the acceptance rate.

H1 regarding performance expectancy did not show a significant, positive effect on behavioral intentions toward an autonomous vehicle. In both the UTAUT and UTAUT2 models, as well as in various other studies, performance expectancy has proved to be the strongest predictor [27]. However, the non-significant effect shows that a large proportion of people do not yet have high performance expectations of autonomous driving systems. The lack of practical experience with autonomous vehicles, the unforeseeable market launch and critical media reports could be reasons. Thus, further testing should be conducted with other items to explore the effect of performance expectancy on behavioral intentions in the context of autonomous driving. $\mathrm{H} 2$, which is also a main predictor in the UTAUT2 model, showed a small significant effect and was supported. This indicates that respondents are confident in handling self-driving vehicles.

The perceived loss of driving pleasure was hypothesized to have a significant, negative effect on behavioral intentions toward autonomous vehicles. However, H8 proved to have no effect and therefore was rejected. This result shows that people do not attach much importance to manual driving and perceive the act of driving as more burdensome than fun. The increasing comfort provided by modern driver assistance systems could contribute to this development. The hypothesized negative effect of desire for control was strongly supported. As postulated, the act of handing over complete control with no option to take over manual control is a major area of concern for study respondents. Other than hypothesized in H6, the environmental friendliness of autonomous vehicles does not influence respondents' behavioral intentions. Previous studies have found that people perceive the positive effects of autonomous vehicles on the environment as a significant benefit [3, 26]. Nevertheless, the non-significant effect indicates that though respondents perceive these developments as a benefit of autonomous vehicles, they are not relevant enough to actually influence their usage intentions. Reasons for the non-significant influence of environmental friendliness on the intention to use 
could also be due to the increasingly controversial discussion of the actual environmental added value of electric powertrains. On the one hand, a considerable part of today's electricity is still being generated from lignite. On the other hand, the carbon dioxide emissions in the production of these cars are often higher than those of conventional cars. Furthermore, a recent study on the tradeoff between increasing environmental impacts of autonomous driving and the expected increases in driving efficiency revealed that autonomous vehicles will result in $9 \%$ reduction in energy and greenhouse gas emissions [8].

The finding that safety has the strongest positive effect on people's intention to use reflects findings of previous studies and supports H9. The safety of an autonomous driving system has top priority for potential users and is a key predictor of their intention to use.

The vehicle security of autonomous vehicles also has a significant, positive effect on people's intention to use. Cyber security and protection against unauthorized access are fundamental requirements for many participants and strongly correlate with the intention to use. Although H11 was accepted, there was only a moderately significant, positive effect. The recent introduction of the general data protection regulation by the EU could have influenced the perception of data privacy. The new legislation provides comprehensive regulations for the privacy and processing of personal data [7].

The postulated positive effect of legal regulations on behavioral intentions was not significant. The rejection of $\mathrm{H} 12$ could be due to the regulation introduced in 2017 of highly and fully automated driving. By introducing this legislation, Germany became the first country to regulate automated driving in a uniform framework. In general, the German Highway Traffic Code is considered safe and reliable. Therefore, people might assume that there will be comprehensive regulations for the market launch of autonomous driving systems.

\section{Conclusions}

The core aim of this paper was to explore the factors that influence the intention to use an autonomous driving system in the future. Therefore, an adapted version of the UTAUT model was introduced.

The empirical findings proved that safety has the strongest positive effect on the intention to use an autonomous vehicle, followed by hedonic motivations. This means that users who assume a high degree of safety aspects are more likely to use an autonomous vehicle than people who assume a low degree of safety. This also means that these people believe that it would be fun to drive an autonomous vehicle in comparison with people who assume a low degree of safety. Furthermore, the desire for control has the strongest negative effect on users' behavioral intentions. That is, users with a high desire for control are less willing to use an autonomous vehicle than people with a low desire for control. Significant, positive effects were also found for effort expectancy, social influence, price-value, safety, security and data privacy. Surprisingly, the performance expectancy construct did not have a significant effect on behavioral intentions. Loss of driving pleasure, environmental friendliness and legal regulations also did not show any significant effects.

The finding that the driving mode received the highest approval shows that the confidence in autonomous driving systems is still expandable. The level of approval of the family mode and the preference for activities that fall into the communication and entertainment categories show that interior design should initially go in this direction. Although the failed activities and concepts that fall into categories such as wellness have received scant attention, it is likely that this could change with increasing experience with autonomous vehicles and confidence.

\section{Future Research}

Future studies could investigate the influencing factors of the acceptance of autonomous driving systems using experiments. Because the actual interaction with autonomous driving systems or, at least, with simulators will provide more comprehensive insights, data should be collected both in advance and after conducting the experiment.

In addition, use of longitudinal studies instead of cross-sectional studies will provide further conclusions about the acceptance factors and their changes. Furthermore, the implementation of cross-cultural studies is recommended in view of the range of this technical development. When assessing moderating effects, research should also ensure that the sample size is sufficient to derive valid results. In general, it is advisable to conduct further testing with larger sample sizes.

Moreover, the involvement of people usually excluded from the use of individual mobility vehicles makes sense, as autonomous driving allows all people, regardless of whether they have a driver's license or physical restrictions, to use autonomous vehicles. For this, further analysis should include moderating factors more extensively.

Finally, the service potential for non-driving-related activities in autonomous vehicles is tremendous, and 
therefore a more intensive investigation of this topic is recommended. As providers' key differentiator is service, the detailed and constant monitoring of preferred activities and their changes is crucial to ensure success.

\section{References}

[1] Antsaklis, P.J., K.M. Passino, and S.J. Wang, "An introduction to autonomous control systems", IEEE Control Systems 11(4), 1991, pp. 5-13.

[2] Bahmanziari, T., J.M. Pearson, and L. Crosby, "Is Trust Important in Technology Adoption? A Policy Capturing Approach", Journal of Computer Information Systems 43(4), 2003, pp. 46-54.

[3] Bansal, P., K.M. Kockelman, and A. Singh, "Assessing public opinions of and interest in new vehicle technologies: An Austin perspective", Transportation Research Part C: Emerging Technologies 67, 2016, pp. 1-14.

[4] Bazilinskyy, P., M. Kyriakidis, and J. de Winter, “An International Crowdsourcing Study into People's Statements on Fully Automated Driving", Procedia Manufacturing 3, 2015, pp. 2534-2542.

[5] Benleulmi, A.Z., and T. Blecker, "Investigating the Factors Influencing the Acceptance of Fully Autonomous Cars", Proceedings of the Hamburg International Conference of Logistics (HICL), epubli (2017), 99-115. [6] Choi, J.K., and Y.G. Ji, "Investigating the Importance of Trust on Adopting an Autonomous Vehicle", International Journal of Human-Computer Interaction 31(10), 2015, pp. 692-702.

[7] European Union, Regulation 2016/679 of the European parliament and the Council of the European Union, 2016.

[8] Gawron, J.H., G.A. Keoleian, R.D. De Kleine, T.J. Wallington, and H.C. Kim, "Life Cycle Assessment of Connected and Automated Vehicles: Sensing and Computing Subsystem and Vehicle Level Effects", Environmental Science and Technology 52(5), 2018, pp. 3249-3256. [9] Hair, J.F., T.G. Hult M., C. Ringle, and M. Sarstedt, $A$ primer on partial least squares structural equation modeling (PLS-SEM), SAGE Publications, Thousand Oaks, CA, 2016. [10] Hein, D., P. Rauschnabel, J. He, L. Richter, and B. Ivens, "What Drives the Adoption of Autonomous Cars?", ICIS 2018 Proceedings, (2018), 17.

[11] Henseler, J., C.M. Ringle, and M. Sarstedt, Using Partial Least Squares Path Modeling in International Advertising Research: Basic Concepts and Recent Issues, Edward Elgar Publishing, Cheltenham, 2012.

[12] Henseler, J., C.M. Ringle, and M. Sarstedt, "A new criterion for assessing discriminant validity in variance-based structural equation modeling", Journal of the Academy of Marketing Science 43(1), 2015, pp. 115-135.

[13] Jo, K., J. Kim, D. Kim, C. Jang, and M. Sunwoo, "Development of Autonomous Car-Part I: Distributed System Architecture and Development Process", IEEE Transactions on Industrial Electronics 61(12), 2014, pp. 7131-7140.

[14] Kung, L.-C., and C.-C. Lin, "Autonomous vehicle services or ride-sharing services? A game theoretic investigation", PACIS 2018 Proceedings, (2018), 267. [15] Kyriakidis, M., R. Happee, and J.C.F. De Winter, "Public opinion on automated driving : Results of an international questionnaire among 5000 respondents", Transportation Research Part F: Psychology and Behaviour 32, 2015, pp. 127-140.

[16] Litman, T., "Autonomous Vehicle Implementation Predictions Implications for Transport Planning”, 2018, 1. www.vtpi.orgInfo@vtpi.org

[17] Madigan, R., T. Louw, M. Wilbrink, A. Schieben, and N. Merat, "What influences the decision to use automated public transport? Using UTAUT to understand public acceptance of automated road transport systems", Transportation Research Part F: Traffic Psychology and Behaviour 50, 2017, pp. 55-64.

[18] Mayer, R.C., J.H. Davis, and F.D. Schoorman, “An Integrative Model Of Organizational Trust", Academy of Management Review 20(3), 1995, pp. 709-734.

[19] Nordhoff, S., J. de Winter, M. Kyriakidis, B. van Arem, and R. Happee, "Acceptance of Driverless Vehicles: Results from a Large Cross-National Questionnaire Study", Journal of Advanced Transportation 2018, 2018, pp. 1-22.

[20] Oldenburg, R., Celebrating the third place: Inspiring stories about the great good places at the heart of our communities, Boston, 2001.

[21] Osswald, S., D. Wurhofer, S. Trösterer, E. Beck, and M. Tscheligi, "Predicting Information Technology Usage in the Car: Towards a Car Technology Acceptance Model", Proceedings of the 4th International Conference on Automotive User Interfaces and Interactive Vehicular Applications - AutomotiveUI '12, ACM Press (2012), 51.

[22] Ostern, N., A. Eßer, and P. Buxmann, "Capturing Users' Privacy Expectations To Design Better Smart Car Applications", PACIS 2018 Proceedings, (2018), 97. [23] Payre, W., J. Cestac, and P. Delhomme, "Intention to use a fully automated car: Attitudes and a priori acceptability", Transportation Research Part F: Traffic Psychology and Behaviour 27, 2014, pp. 252-263. [24] Rödel, C., S. Stadler, A. Meschtscherjakov, and M. Tscheligi, "Towards Autonomous Cars: The Effect of Autonomy Levels on Acceptance and User Experience", Proceedings of the 6th International Conference on Automotive User Interfaces and Interactive Vehicular Applications, ACM Press (2014), 1-8.

[25] SAE International, J3016B: Taxonomy and Definitions for Terms Related to Driving Automation Systems for OnRoad Motor Vehicles - SAE International, 35, 2018. [26] Schoettle, B., and M. Sivak, A survey of public opinion about autonomous and self-driving vehicles in the U.S., the U.K., and Australia, University of Michigan, Ann Arbor, Michigan, 2014.

[27] Venkatesh, V., J.Y. L. Thong, and X. Xu, "Consumer acceptance and use of information technology: extending the unified theory of acceptance and use of technology", MIS Quarterly 36(1), 2012, pp. 157-178.

[28] Wu, K., Y. Zhao, Q. Zhu, X. Tan, and H. Zheng, “A meta-analysis of the impact of trust on technology acceptance model: Investigation of moderating influence of subject and context type", International Journal of Information Management 31(6), 2011, pp. 572-581. 